Ann. Biol. anim. Bioch. Biophys., 1977, 17 (4), 549-552.

\title{
Effet des proanthocyanidines extractibles au méthanol absolv (PEMA) sur la digestibilité des protéines chez le poussin (essai préliminaire)
}

\author{
par J. GUILLAUME, Joëlle GOMEZ
}

Station de Recherches Avicoles, I.N.R.A., Nouzilly, 37380 Monnaie.

Summary. Effect of proanthocyanidins extracted with absolute methanol (PEMA) on protein digestibility in chick (Preliminary results).

Recent studies (Martin-Tanguy ef al., 1976) showed that field bean (Vicia faba var. minor) contains variable quantities of extracted with absolute methanol proanthocyanidins (PEMA) depending on the genetic origin of the plant. PEMA effect was measured after protein precipitation in the faeces. When the level of field bean proteins varied from 34 up to 100 p. 100 , of total protein, nitrogen digestibility decreased from 87 to 77 p. 100 when field bean was raw, and from 87 to 82 p. 100 when it was heated. Moreover, independently of heating, there was a linear relationship between nitrogen digestibility and level of horse bean proteins. PEMA which were almost completely destroyed by heating might be components of the antinutritional factor emphasized in Vicia faba.

L'influence des facteurs exogènes sur la digestibilité des éléments nutritifs est mal connue chez la volaille. Chez le Poulet, nous avons constaté que les protéines de féverole (Vicia faba $L$ ) étaient moins bien digérées que cєlles de plantes botaniquement voisines, soja et pois (Guillaume et Bellec, 1975). Le facteur antitrypsique abondant dans le soja n'est qu'en concentration réduite dans la féverole (Wilson et Mc Nab, 1972), on peut difficilement admettre qu'il soit responsable de l'effet dépressif observé. Les tannins présents dans la féverole (Baker, et Morris, 1968) constituent un ensemble de composés auxquels on pouvait, a priori, attribuer des effets défavorables à l'utilisation digestive des protéines. Récemment, Martin-Tanguy, Guillaume et Kossa (1977) ont montré que ces tannins étaient des proanthocyanidines polymères de flavanols 3 (catéchine et gallocatéchine) et flavanediols 3-4 (leucocyanidine, leucodelphinidine). Certaines de ces proanthocyanidines, les fractions très condensées extractibles au méthanol absolu (qui sont en concentration variable selon les variétés), semblent responsables de la diminution du coefficient d'utilisation digestive apparent des protéines $\left(C U D_{a} N\right)$ de féverole : il existe une corrélation négative significative entre les fractions de proanthocyanidines extractibles au méthanol absolu (PEMA) et le CUD $a$ N. Il semble exister également une liaison négative entre la teneur en PEMA et le poids moyen de l'œuf chez des poules nourries avec des régimes contenant 30 p. 100 de féverole (Guillaume et Bellec, 1977).

Etant, donné la fragilité des molécules de PEMA aux températures élevées, 
nous avons recherché si l'effet apparent des PEMA sur l'utilisation digestive des protéines ne pouvait pas être réduit par autoclavage de la graine. Nous rapportons ici les résultats de deux des essais préliminaires entrepris dans ce but.

\section{Matériel et méthodes.}

Comme il est difficile d'extraire les PEMA sans altération de leur structure, nous avons travaillé sur des féveroles de trois origines distinctes : la lignée S45 très riche en PEMA, le cultivar Bianka dépourvu de PEMA mais riche en phénols non condensés et la lignée « Zéro Tannin » $\left(^{1}\right)$ également dépourvue de PEMA et plus pauvre en phénols non condensés $\left(^{1}\right)$. Le traitement thermique utilisé est un autoclavage de la graine à $120^{\circ} \mathrm{C}$ pendant $30 \mathrm{mn}$.

\section{TABLEAU 1}

Composition des régimes de base (p. 100)

Régime poisson-gluten

Farine de poisson 65 p. 100 prot. ..........

Gluten de maïs 60 p. 100 prot. ............

Glucose monohydrate.................

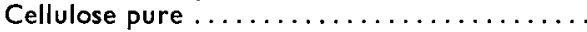

L lysine $\mathrm{HCl} \ldots \ldots \ldots \ldots \ldots \ldots \ldots \ldots \ldots \ldots$

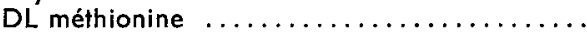

Prémélange minéral et vitaminique $\ldots \ldots \ldots \ldots$

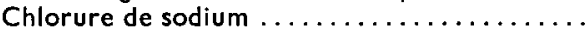

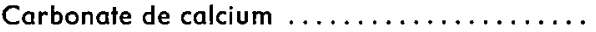

Régimes féverole

Féverole $\$ 45 \ldots \ldots \ldots \ldots \ldots \ldots \ldots \ldots \ldots \ldots$

Féverole Bianka ou Zéro Tannin...........

Glucose monohydrate.................

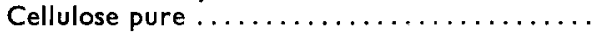

Huile de maïs $\ldots \ldots \ldots \ldots \ldots \ldots \ldots \ldots \ldots$

Vermiculite $\ldots \ldots \ldots \ldots \ldots \ldots \ldots \ldots \ldots \ldots$

DL-méthionine $\ldots \ldots \ldots \ldots \ldots \ldots \ldots \ldots$

Prémélange minéral ef vitaminique $\ldots \ldots \ldots \ldots$

Chlorure de sodium . . . . . . . . . . . . .

Phosphate bicalcique ................

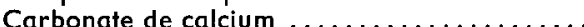

Caractéristiques principales des régimes

Energie métabolisable, $\mathrm{Kcal} / \mathrm{g} \ldots \ldots \ldots \ldots \ldots$

Protéines totales $(\mathrm{N} \times 6,25)(\mathrm{p} .100) \ldots \ldots \ldots$

Lysine $(($ p. 100) . . . . . . . . . . . . . . . .

Acides aminés soufrés (p. 100) ............

Calcium (p. 100) ....................

Phosphore disponible (p. 100)
17,5

17,5

56

7

0,25

0,10

0,70

0,30

0,65

\section{S 45 Bianka ou}

Zéro Tannin

85

$\begin{array}{ll}- & 80,5 \\ = & 3,5 \\ 9 & 1 \\ 1,25 & 9 \\ 0,35 & 1,25 \\ 0,70 & 0,35 \\ 0,30 & 0,70 \\ 2,20 & 0,30 \\ 1,20 & 2,20 \\ & 1,20\end{array}$

3,02

22,4

$>1,25$

0,95

1,10

0,50

(1) Tous ces échantillons sont fournis par la Station d'Amélioration des Plantes de I'I.N.R.A., BV 1540, 21034 Dijon Cedex. Les indications sur les teneurs en PEMA et phénols non condensés nous sont fournies par les chercheurs de cette Station. 
Nous avons consłitué d'abord trois mélanges de base de caractéristiques nutritionnelles aussi voisines que possible où la seule source de protéines éłaił respectivement (tabl. 1) : un mélange farine de poisson-gluten de maïs, la féverole $\$ 45$ crue ou autoclavée, la féverole Bianka crue ou autoclavée dans l'essai 1, la féverole Zéro Tannin crue ou autoclavée dans l'essai 2 (tabl. 2). Ces mélanges de base ont été ensuite combinés de façon à obtenir 16 régimes définitifs où le pourcentage de protéines provenant de la féverole varie de 0 à 100 p. 100 aux dépens de la protéine du mélange poisson-gluten très digestible.

TABLEAU 2

Coefficients de digestibilité apparenfe de l'azote des divers régimes

\begin{tabular}{|c|c|c|c|c|c|}
\hline Essai 1 & Aufoclavage & $34^{N}$ & P. 1 & $\begin{array}{c}00 \mathrm{NT} \\
66\end{array}$ & * 100 \\
\hline \multirow{2}{*}{$\begin{array}{l}\text { Riche en tannins } \ldots \ldots \ldots \ldots \ldots \\
\text { (S 45) }\end{array}$} & 一 & 87,2 & - & 82,8 & 77,4 \\
\hline & $120^{\circ} \mathrm{C} \quad 30 \mathrm{mn}$ & 87,4 & - & 84,7 & 82,1 \\
\hline \multirow{2}{*}{$\begin{array}{l}\text { Sans tannins } \\
\text { (Bianka) }\end{array}$} & - & 89,4 & - & 89,1 & 81,1 \\
\hline & $120^{\circ} \mathrm{C} \quad 30 \mathrm{mn}$ & - & 88,8 & - & 88,1 \\
\hline Essai 2 & Autoclavage & \multicolumn{3}{|c|}{$\begin{array}{llr}\text { NF p. } 100 & N^{*} \\
0 & 50 & 100\end{array}$} & \\
\hline \multirow{2}{*}{$\begin{array}{l}\text { Sans tannins } \ldots \ldots \cdots \cdots \cdots \\
\text { (Zéro Tannin) }\end{array}$} & - & \multirow{2}{*}{86,2} & 83,8 & 81,9 & \\
\hline & $120^{\circ} \mathrm{C} \quad 30 \mathrm{mn}$ & & 84,7 & 82,1 & \\
\hline
\end{tabular}

* $\mathrm{N}$ de la féverole p. 100 de $\mathrm{N}$ total du régime.

Chaque régime a été distribué pendant 5 jours à 2 groupes de 3 poussins élevés en cages individuelles. Pendant les 3 derniers jours on a effectué un bilan sur chaque groupe de 3 animaux. On a mesuré la digestibilité apparente des protéines par précipitation des protéines fécales à l'acétate de plomb selon Terpstra et De Hart, (1974).

\section{Résultats et discussion.}

Les résultats de cet essai sont rassemblés dans le tableau 2. Ils sont en accord avec nos résultats antérieurs puisque la digestibilité de l'azote est nettement plus faible dans la féverole riche en tannins que dans les féveroles dépourvues de PEMA, du moins dans les régimes contenant des pourcentages élevés de cette légumineuse. Un point nouveau apparaît : l'autoclavage de la féverole améliore de façon très nette la digestibilité des protéines de la féverole riche en tannins alors qu'il est sans effet sur la féverole Zéro Tannin et n'agit, de façon également bénéfique, sur les 
protéines de la variété Bianka que si celles-ci représentent 100 p. 100 des protéines de la ration.

On peut également noter que la liaison entre $\mathrm{CUD}_{\mathrm{a}} \mathrm{N}$ et pourcentage de féverole n'est linéaire que dans le cas de la féverole Zéro Tannin crue et dans le cas des féveroles autoclavées.

Nous avons vérifié par ailleurs que l'autoclavage à $120^{\circ} \mathrm{C}$ pendant $30 \mathrm{mn}$ détruit presque totalement les PEMA. Celles-ci, en revanche, se retrouvent abondamment dans les feces des poussins ayant ingéré de la féverole $S 45$ crue (elles sont alors sous forme complexée).

Une partie des résultats reste obscure : la protéine de féverole Zéro Tannin semble moins digestible que celle de la féverole Bianka. Signalons toutefois que l'origine culturale des échantillons n'était pas la même et que les mesures ont été faites dans deux essais distincts.

Les résultats apportent cependant de nouveaux éléments concernant le rôle antinutritionnel de certains tannins contenus dans la féverole. Ils permettent aussi de formuler l'hypothèse selon laquelle les PEMA constituent l'un des facteurs antinutritionnels thermolabiles dont l'existence a été récemment mise en évidence dans la féverole par Marquardt, Campbell et Ward (1976). Ils n'apportent toutefois aucune indication sur le mode d'action de ces composés. II est vraisemblable que la formation de complexes stables entre PEMA ef protéines alimentaires est responsable de la mauvaise utilisation digestive de ces dernières, la destruction par la chaleur des PEMA rendant la formation de complexes impossible. Il se peut qu'une partie de ces réactions se produise pendant le chauffage lui-même, ce qui expliquerait que la protéine de la lignée S45 reste de toute façon moins digestible que celle du cultivar Bianka. Par ailleurs, une influence éventuelle des phénols non condensés sur la digestibilité, pourraił expliquer la chute du $\operatorname{CUD}_{a} \mathrm{~N}$ dans le régime où 100 p. 100 des protéines proviennent de la variété Bianka (mais non de la lignée Zéro Tannin). Enfin, un effet des PEMA sur la digestibilité d'autres éléments nutritifs n'esł pas à exclure. D'autres travaux sont donc nécessaires pour élucider le rôle des proanthocyanidines extractibles au méthanol absolu sur la digestibilité des nutriments.

Commission CNERNA Digestion-Absorption, Tours, 13 novembre 1976.

\section{Références}

BAKER P. A., MORRIS D. J., 1968. Leucoanthocyanidines in the broad bean. Sch. Sci. Rev., 49, 79-86. GUILLAUME J., BELLEC R., 1975. Données non publiées.

GUILLAUME J., BELLEC R., 1977. Use of field beans (Vicia faba L.) in laying hen feeds. Brit. Poult. Sci. (sous presse).

MARQUARDT R. R., CAMPBELL L. D., WARD T., 1976. Studies with chicks on the growth depressing factor(s) in Faba beans (Vicia faba L. var. minor). J. Nutr., 106, 275-284.

MARTIN-TANGUY J., GUILLAUME J., KOSSA A., 1977. Condensed tannins in horse bean seeds : chemical structure and effects on the food value of the horse bean in growing poultry. $J$ Sci. Food agric. (sous presse).

TERPSTRA K., DE HART N., 1974. The estimation of urinary nitrogen and fecal nitrogen in poultry excreta. Z. Tierphysiol. Tierernaehr. Futtermittelkd., 32, 306-320.

WILSON B. J., MC NAB J. M., 1972. The effect of autoclaving and methionine supplementation on the growth of chicks given diets containing field beans. Brit. Poult. Sci., 13, 67-73. 